\title{
Effective Lagrangians on Domain Walls and Other Solitons
}

\section{Norisuke Sakai ${ }^{* \dagger}$}

Department of Physics, Tokyo Institute of Technology, Tokyo 152-8551, Japan

E-mail: sakai.n.aa(at)m.titech.ac.jp

\section{Minoru Eto}

University of Tokyo, Inst. of Physics, Komaba 3-8-1, Meguro-ku Tokyo 153, Japan

E-mail: meto (at) hep1.c.u-tokyo.ac.jp

\section{Youichi Isozumi}

Department of Physics, Tokyo Institute of Technology, Tokyo 152-8551, Japan

E-mail: isozumi.y.aa(at)m.titech.ac.jp

\section{Muneto Nitta}

Department of Physics, Keio University, Hiyoshi, Yokohama, Kanagawa 223-8521, Japan

E-mail: nitta (at) phys-h.keio.ac.jp

\section{Keisuke Ohashi}

Department of Physics, Tokyo Institute of Technology, Tokyo 152-8551, Japan

E-mail: keisuke (at)th.phys.titech.ac.jp

\begin{abstract}
Maintaining the preserved supersymmetry helps to find the effective Lagrangian on the BPS background in gauge theories with eight supercharges. As concrete examples, we take 1/2 BPS domain walls. The Lagrangian is given in terms of the superfields with manifest four preserved supercharges and is expanded in powers of the slow-movement parameter $\lambda$. The $\mathscr{O}\left(\lambda^{0}\right)$ gives the superfield form of the BPS equations, whereas all the fluctuation fields follow at $\mathscr{O}\left(\lambda^{1}\right)$. The effective Lagrangian is given by the density of the Kähler potential which emerges automatically from the $\lambda$ expansion making four preserved supercharges manifest. More complete account of our method and applications is given in [1] (hep-th/0602289) in which the case of non-Abelian vortices is also worked out.
\end{abstract}

From Strings to LHC

January 2-10 2007

International Centre Dona Paula, Goa India

\footnotetext{
*Speaker.

$\dagger$ Partly supported by Grant-in-Aid for Scientific Research from the Ministry of Education, Culture, Sports, Science and Technology, Japan No.17540237and No.18204024.
} 


\section{Introduction}

In the brane-world scenario, our four-dimensional spacetime is realized as a topological defect in a higher dimensional spactime [2]. It is desirable to construct such topological defect as various topological solitons. Supersymmetric gauge theories have been extremely useful to construct realistic models beyond the standard model [3]. When a field configuration preserves a part of supersymmetry (SUSY), it satisfies the field equation automatically [4]. Such a field configuration is called the Bogomol'nyi-Prasad-Sommerfield (BPS) state [5]. The BPS solitons in the Higgs phase are extensively reviewed recently [6]. Quite often BPS solitons contain a number of parameters such as positions in the space-time and/or an internal space. These parameters are called moduli. To understand the dynamics of solitons for the brane-world scenario, it is important to construct the low-energy effective Lagrangian of the localized modes on such solitons. For that purpose, the standard method is to promote the moduli parameters of the background soliton into fields on the world volume of the soliton [7]. The moduli fields provide massless fields on the world volume of the soliton. This moduli approximation method is based on the assumption of the weak dependence on the world-volume coordinates, and gives the low-energy effective Lagrangian which contains all nonlinear terms with two derivatives or less. We have recently worked out a systematic method to obtain the effective Lagrangian on the BPS background in supersymmetric gauge theories, taking domain walls and vortices as concrete examples [1]. We have introduced a slow-movement parameter $\lambda$, and expanded the Lagrangian in terms of the superfields with four preserved supercharges in powers of the slow-movement parameter. We have found that maintaining the preserved supersymmetry manifest facilitates the procedure enormously. In this article, we introduce the systematic method taking domain walls as an example.

We consider the supersymmetric $U\left(N_{\mathrm{C}}\right)$ gauge theories with eight supercharges with $N_{\mathrm{F}}\left(\geq N_{\mathrm{C}}\right)$ hypermultiplets in the fundamental representation as an illustrative example. Although we work in the space-time dimensions highest allowed by supersymmetry, namely domain walls in five dimensions, our discussion should be applicable in lower dimensions which can be obtained by dimensional reductions. We can naturally specify the order of magnitude in powers of the slowmovement parameter $\lambda$ for various fields. Thus we obtain a systematic expansion of the Lagrangian in powers of $\lambda$. The expansion gives a superfield form of the BPS equations at the zero-th order in $\lambda$, and the superfield equation to determine all the fluctuation fields at the next order. We retain up to the terms of order $\lambda^{2}$ in the Lagrangian, in order to obtain the effective Lagrangian at the lowest nontrivial order, namely up to two derivatives. We are now computing the higher powers of $\lambda$ in our systematic expansion to obtain the effective Lagrangian with higher derivative terms. We maintain four preserved SUSY manifest throughout our procedure, and obtain a density of the Kähler potential in four SUSY superspace. By integrating over the extra dimensions, we obtain the Kähler potential of the effective Lagrangian which was difficult to obtain in general previously. Our results can be used to study soliton scattering in $U\left(N_{\mathrm{C}}\right)$ gauge theories.

\section{Slow-move Approximation in terms of Component Fields}

Let us introduce our model and describe briefly the usual component method to solve the BPS equations. The bosonic parts of the Lagrangian with a common gauge coupling constant $g$ for 
$U\left(N_{\mathrm{C}}\right)=S U\left(N_{\mathrm{C}}\right) \times U(1)$ in five dimensions is given by

$$
\left.\mathscr{L}\right|_{\text {boson }}=\operatorname{Tr}\left[-\frac{1}{2 g^{2}} F_{M N}(W) F^{M N}(W)-\frac{1}{g^{2}}\left(\mathscr{D}_{M} \Sigma\right)^{2}-\mathscr{D}^{M} H^{i}\left(\mathscr{D}_{M} H^{i}\right)^{\dagger}\right]-V .
$$

The physical bosonic components in the vector multiplet are gauge fields $W_{M}$, and the real scalar fields $\Sigma$ in the adjoint representation, and those in the hypermultiplet are the doublets of the complex scalar fields $H^{i} i=1,2$ which can be assembled into $N_{\mathrm{C}} \times N_{\mathrm{F}}$ matrices. The indices $M, N=0,1, \cdots, 4$ run over five-dimensions, and the mostly plus signature is used for the metric $\eta_{M N}=$ diag. $(-1,+1, \cdots,+1)$. The covariant derivatives are defined as $D_{M} \Sigma=\partial_{M} \Sigma+i\left[W_{M}, \Sigma\right]$, $D_{M} H^{i}=\left(\partial_{M}+i W_{M}\right) H^{i}$, and field strength is defined as $F_{M N}=\frac{1}{i}\left[D_{M}, D_{N}\right]=\partial_{M} W_{N}-\partial_{N} W_{M}+$ $i\left[W_{M}, W_{N}\right]$. After eliminating auxiliary fields, the scalar potential $V$ is given by

$$
V=\frac{g^{2}}{4} \operatorname{Tr}\left[\left(H^{1} H^{1 \dagger}-H^{2} H^{2 \dagger}-c \mathbf{1}_{N_{\mathrm{C}}}\right)^{2}+4 H^{2} H^{1 \dagger} H^{1} H^{2 \dagger}\right]+\operatorname{Tr}\left[\left(\Sigma H^{i}-H^{i} M\right)\left(\Sigma H^{i}-H^{i} M\right)^{\dagger}\right]
$$

with the hypermultiplet mass matrix $M=\operatorname{diag}\left(m_{1}, \cdots, m_{N_{\mathrm{F}}}\right)\left(m_{A} \in \mathbf{R}\right)$ and the Fayet-Iliopoulos parameter taken along the third direction in $S U(2)_{R}$ as $c_{a}=(0,0, c)$ with $c>0$.

By requiring half of SUSY to be preserved, we obtain the $1 / 2$ BPS equations for domain walls which depend on $y$ only

$$
\begin{aligned}
\mathscr{D}_{y} H^{1} & =-\Sigma H^{1}+H^{1} M, \quad \mathscr{D}_{y} H^{2}=\Sigma H^{2}-H^{2} M, \\
\mathscr{D}_{y} \Sigma & =\frac{g^{2}}{2}\left(c \mathbf{1}_{N_{\mathrm{C}}}-H^{1} H^{1 \dagger}+H^{2} H^{2 \dagger}\right), \quad 0=g^{2} H^{1} H^{2 \dagger} .
\end{aligned}
$$

The solution of the BPS equations saturates the BPS bound for the tension of the (multi-)wall

$$
\begin{aligned}
T_{\mathrm{w}} & =\int_{-\infty}^{+\infty} d y \mathscr{E}_{\mathrm{w}}=\int_{-\infty}^{+\infty} d y \partial_{y}\left[\operatorname{Tr}\left[c \Sigma-\left(\Sigma H^{1} H^{1 \dagger}-H^{1} M H^{1 \dagger}\right)+\left(\Sigma H^{2} H^{2 \dagger}-H^{2} M H^{2 \dagger}\right)\right]\right] \\
& =c[\operatorname{Tr} \Sigma]_{-\infty}^{+\infty}
\end{aligned}
$$

where the energy density is denoted as $\mathscr{E}_{\mathrm{w}}$. We can solve the hypermultiplet BPS equation (2.3) by introducing an $N_{\mathrm{C}} \times N_{\mathrm{F}}$ constant matrix $H_{0}$ called the moduli matrix [8], [9]

$$
\begin{aligned}
& \Sigma+i W_{y}=S^{-1}(y) \partial_{y} S(y), \quad W_{\mu}=0, \quad(\mu=0, \cdots, 3) \\
& H^{1}=S^{-1}(y) H_{0} e^{M y}, \quad H^{2}=0,
\end{aligned}
$$

where the moduli matrix $H_{0}$ carries all the parameters of the solution, namely moduli. The moduli matrices related by the following $V$-equivalence transformations are physically equivalent:

$$
H_{0} \rightarrow V H_{0}, \quad S(y) \rightarrow V S(y), \quad V \in G L\left(N_{\mathrm{C}}, \mathbf{C}\right) .
$$

The vector multiplet BPS equation (2.4) can be converted to the following "master equation" for a gauge invariant quantity $\Omega \equiv S S^{\dagger}[8$

$$
\partial_{y}\left(\Omega^{-1} \partial_{y} \Omega\right)=g^{2} c\left(\mathbf{1}_{N_{\mathrm{C}}}-\Omega^{-1} \Omega_{0}\right), \quad \Omega_{0} \equiv c^{-1} H_{0} e^{2 M y} H_{0}^{\dagger} .
$$

The matrix function $S$ can be determined from the solution $\Omega$ of this master equation by fixing a gauge, and all the other fields can be obtained from $S$ and $H_{0}$. Since the BPS soliton has codimension one, the solution represents (multiple parallel) domain walls. 
We can obtain the low-energy effective Lagrangian by promoting the moduli parameters $\phi^{\alpha}$ in the moduli matrix $H_{0}$ to fields on the soliton world volume depending on $x^{\mu},(\mu=0,1,2,3)$

$$
H_{0}\left(\phi^{\alpha}\right) \rightarrow H_{0}\left(\phi^{\alpha}(x)\right) .
$$

To represent the weak dependence on the world-volume coordinates of the soliton [7], we introduce "the slow-movement parameter" $\lambda$, which is assumed to be much smaller than the typical mass scale in the problem, in our case, the hypermultiplet mass difference $\Delta m$ and $g \sqrt{c}$ where $c$ and $g$ are the Fayet-Iliopoulos parameter and the gauge coupling.

$$
\lambda \ll \min (\Delta m, g \sqrt{c}) .
$$

The nonvanishing fields of the $1 / 2$ BPS background have contributions independent of $\lambda$, and derivatives in terms of the world volume coordinates are assumed to be of order $\lambda$, expressing the weak dependence on the world-volume coordinates

$$
H^{1} \sim \mathscr{O}(1), \quad \Sigma \sim \mathscr{O}(1), \quad \partial_{\mu} \sim \mathscr{O}(\lambda) .
$$

Those fields which vanish in the background solution can now have nonvanishing values, induced by the fluctuations of the moduli fields of order $\lambda$

$$
\begin{gathered}
W_{\mu} \sim \mathscr{O}(\lambda), \quad H^{2} \sim \mathscr{O}(\lambda), \\
\mathscr{D}_{\mu} H^{1} \sim \mathscr{O}(\lambda), \quad \mathscr{D}_{\mu} \Sigma \sim \mathscr{O}(\lambda), \quad F_{\mu y}(W) \sim \mathscr{O}(\lambda),
\end{gathered}
$$

and other components of the field strength are higher orders in $\lambda$. If we decompose the field equations in powers of $\lambda$, we find that order $\lambda^{0}$ equations are automatically satisfied by the BPS equations (2.3) and (2.4). However, it becomes more and more complicated to solve the field equation at higher orders in the expansion in powers of $\lambda$, since various fields that vanish in the background become nonvanishing, and need to be solved. We shall show in the next section that maintaining the preserved SUSY manifest greatly helps to determine these newly nonvanishing fields and to organize the expansion of field equations in powers of $\lambda$.

\section{Slow-move Approximation in terms of Superfields}

Since four supercharges are conserved by the BPS domain walls, an action for fluctuations around the BPS background can be written in term of the superfield for four supercharges. Let us define the superfields ${ }^{1}$ using two component spinor Grassmann coordinates $\theta^{\alpha}, \bar{\theta}_{\dot{\alpha}}$. The components of superfields are fields in five dimensions. A vector multiplet with eight SUSY consists of a real vector superfield $\mathbf{V}\left(=\mathbf{V}^{\dagger}\right)$ and an adjoint chiral superfield $\Phi\left(\bar{D}_{\dot{\alpha}} \Phi=0\right)$ in terms of superfield with four supercharges [13]. The vector superfield $\mathbf{V}$ contains a gauge field $W_{\mu}, \mu=0, \cdots, 3$ for the four spacetime dimensions, the half of gaugino field $\lambda_{+}$, and an auxiliary field $\mathscr{Y}^{3}$. If one takes the Wess-Zumino gauge, it becomes explicitly as

$$
\left.\mathbf{V}\right|_{\mathrm{WZ}}=-\theta \sigma^{\mu} \bar{\theta} W_{\mu}+i \theta^{2} \bar{\theta} \bar{\lambda}_{+}-i \bar{\theta}^{2} \theta \lambda_{+}+\frac{1}{2} \theta^{2} \bar{\theta}^{2} \mathscr{Y}^{3}, \quad \mathscr{Y}^{3} \equiv Y^{3}-\mathscr{D}_{y} \Sigma,
$$

\footnotetext{
${ }^{1}$ We use the convention of Wess and Bagger [10] for Grassmann coordinates and superfields in this paper, except that four-dimensional spacetime indices are denoted by Greek alphabets $\mu, v=0, \cdots, 3$. For conventions of superfields in terms of component fields, we mostly follow those in Refs.[11], and [12].
} 
where the auxiliary field $\mathscr{Y}^{3}$ of the superfield for four SUSY is shifted from the auxiliary field $Y^{3}$ for eight SUSY by the covariant derivative of adjoint scalar $\Sigma$ along the fifth coordinate (the extra dimensions) $y[11]$, [12]. This difference becomes important in identifying the topological charge later. The chiral scalar superfield $\Phi$ contains a complex scalar field made of the adjoint scalar $\Sigma$ and the fifth component of the gauge field $W_{y}$ as the real and imaginary parts respectively, and the other half of gaugino $\lambda_{-}$and a complex auxiliary field $Y^{1}+i Y^{2}$

$$
\Phi=\Sigma+i W_{y}+\sqrt{2} \theta\left(-i \sqrt{2} \lambda_{-}\right)+\theta^{2}\left(Y^{1}+i Y^{2}\right) .
$$

The hypermultiplet are represented by a chiral superfield $\mathbf{H}^{1}$ and an anti-chiral superfield $\mathbf{H}^{2}$. The (anti-) chiral superfield $\mathbf{H}^{1}\left(\mathbf{H}^{2}\right)$ consists of the physical complex scalar field $H^{1}\left(H^{2}\right)$, hyperino $\psi_{+}\left(\psi_{-}\right)$, and a complex auxiliary field $\mathscr{F}^{1}\left(\mathscr{F}^{2}\right)$

$$
\begin{array}{ll}
\mathbf{H}^{1}=H^{1}+\sqrt{2} \theta \psi_{+}+\theta^{2} \mathscr{F}^{1}, & \mathscr{F}^{1} \equiv F^{1}+\left(\mathscr{D}_{y}-\Sigma\right) H^{2}+H^{2} M, \\
\mathbf{H}^{2}=H^{2}+\sqrt{2} \bar{\theta} \bar{\psi}_{-}+\bar{\theta}^{2} \mathscr{F}^{2}, & \mathscr{F}^{2} \equiv-F^{2}-\left(\mathscr{D}_{y}+\Sigma\right) H^{1}+H^{1} M,
\end{array}
$$

where the auxiliary field $\mathscr{F}^{1}\left(\mathscr{F}^{2}\right)$ of the superfield for four SUSY is shifted from the auxiliary field $F^{1}\left(F^{2}\right)$ for eight SUSY by the covariant derivative of the other hypermultiplet scalar $H^{2}\left(H^{1}\right)$ and other ${ }^{2}$ terms [11], [12]. Please note that we have chosen to denote the anti-chiral superfield as $\mathbf{H}^{2}$, as shown in the $\bar{\theta}$ dependence in Eq. (3.4).

The derivative $\hat{D}_{y}$ which is covariant under the complexified gauge transformations for the hypermultiplet $\mathbf{H}^{1}$ and the adjoint chiral scalar multiplet $\Phi$ are given by

$$
\hat{D}_{y} \mathbf{H}^{1}=\left(\partial_{y}+\Phi\right) \mathbf{H}^{1}, \quad \hat{D}_{y} e^{2 \mathbf{V}}=\partial_{y} e^{2 \mathbf{V}}-\Phi^{\dagger} e^{2 \mathbf{V}}-e^{2 \mathbf{V}} \Phi .
$$

If supplemented by fermionic terms, the bosonic Lagrangian 2.10 becomes invariant under the supersymmetric transformations with eight (real) Grassmann parameters. We can now rewrite this fundamental Lagrangian $\mathscr{L}$ in terms of the superfields for four supercharges as

$$
\begin{aligned}
\mathscr{L}= & -\mathscr{E}_{\mathbf{w}}+\int d^{4} \theta \operatorname{Tr}\left[-2 c \mathbf{V}+\frac{1}{2 g^{2}}\left(e^{-2 \mathbf{V}} \hat{D}_{y} e^{2 \mathbf{V}}\right)^{2}+e^{2 \mathbf{V}} \mathbf{H}^{1} \mathbf{H}^{1 \dagger}+e^{-2 \mathbf{V}} \mathbf{H}^{2} \mathbf{H}^{2 \dagger}\right] \\
& +\left(\int d^{2} \theta \operatorname{Tr}\left[\hat{D}_{y} \mathbf{H}^{1} \mathbf{H}^{2 \dagger}-\mathbf{H}^{1} M \mathbf{H}^{2 \dagger}+\frac{1}{4 g^{2}} \mathbf{W}^{\alpha} \mathbf{W}_{\alpha}\right]+\text { h.c. }\right),
\end{aligned}
$$

where field strength superfield $\mathbf{W}$ is given by

$$
\mathbf{W}_{\alpha} \equiv-\frac{1}{8} \bar{D} \bar{D} e^{-2 \mathbf{v}} D_{\alpha} e^{2 \mathbf{v}}
$$

In transforming the fundamental Lagrangian (3.6) in terms of the superfield for four SUSY into the manifestly supersymmetric form for eight SUSY 2.1), we need to make several partial integrations with respect to the fifth coordinate $y$, and have to retain the surface terms carefully in the procedure. We also note that the auxiliary fields for four SUSY $\mathscr{Y}^{3}$, and $\mathscr{F}^{i}$ are different from those for eight SUSY $Y^{3}, F^{i}$ by total derivative terms as in Eqs. 3.17, 3.3), and 3.4). In this way we find a

\footnotetext{
${ }^{2}$ The other terms involving the adjoint scalar $\Sigma$ and the hypermultiplet mass matrix $M$ can be understood as a result of the Scherk-Schwarz dimensional reduction from six dimensions.
} 
total divergence $\mathscr{E}_{\mathrm{w}}$ representing the topological charge contributing to the energy density of the background which maintains four SUSY. Since we are interested in bosonic components of the topological term $\mathscr{E}$, we exhibit only the bosonic terms explicitly

$$
\begin{aligned}
\mathscr{E}_{\mathrm{w}}= & \partial_{y}\left[\operatorname { T r } \left[c \Sigma-\left(\Sigma H^{1} H^{1 \dagger}-H^{1} M H^{1 \dagger}\right)+\left(\Sigma H^{2} H^{2 \dagger}-H^{2} M H^{2 \dagger}\right)\right.\right. \\
& \left.\left.-\frac{2}{g^{2}} \mathscr{Y}^{3} \Sigma+\mathscr{F}^{1} H^{2 \dagger}+H^{2} \mathscr{F}^{1 \dagger}+(\text { fermionic terms })\right]\right] .
\end{aligned}
$$

Let us emphasize again that the topological term is precisely the difference between the fundamental Lagrangian which is manifestly supersymmetric under the eight SUSY and another fundamental Lagrangian in terms of superfields for four manifest SUSY.

Following the usual procedure[7], we promote the moduli $\phi^{i}$ to fields $\phi^{i}(x)$ on the world volume of the background soliton, and assume that the moduli fields $\phi^{i}(x)$ around the wall background to fluctuate only very slowly. Namely, we introduce a parameter $\lambda$ for the slow movement and neglect high energy fluctuations as explained in sect By explicitly writing the derivatives of moduli fields we obtain

$$
\partial_{y} \phi^{i}=\mathscr{O}(1) \phi^{i}, \quad \partial_{\mu} \phi^{i}=\mathscr{O}(\lambda) \phi^{i}, \quad \lambda \ll \min (\Delta m, g \sqrt{c}) .
$$

Here and in the following, $\mathscr{O}(1)$ means that it is of the order of the characteristic mass scale $\min (\Delta m, g \sqrt{c})$. The slow-movement parameter $\lambda$ in Eq. 3.9] is defined to be of the order of the world-volume-coordinate derivative $\partial_{\mu}$. The supertransformation implies that the square of the derivative in terms of the Grassmann coordinates $\theta$ gives translation in the world-volume : $(\partial / \partial \theta)^{2} \sim \partial_{\mu}$. Therefore we obtain $d \theta \sim \partial / \partial \theta \sim \mathscr{O}\left(\lambda^{\frac{1}{2}}\right)$. To assign the order of $\lambda$ for hypermultiplets, we observe that the first hypermultiplet $H^{1}$ has nonvanishing values whereas the second hypermultiplet $H^{2}$ vanishes in the 1/2 BPS background solution 2.77. If we let the moduli parameters to fluctuate over the world-volume coordinates with the order of $\lambda$, the fluctuation induces terms of order $\lambda$ in both hypermultiplets. Therefore the second hypermultiplet $H^{2}$ naturally becomes nonvanishing values and is of order $\lambda$. Combining the above order estimates of component fields, we assume the order of the hypermultiplet superfields and the adjoint chiral scalar superfield

$$
\mathbf{H}^{1} \sim \mathscr{O}(1), \quad \mathbf{H}^{2} \sim \mathscr{O}(\lambda), \quad \Phi \sim \mathscr{O}(1) .
$$

Note that this assignment breaks half of supersymmetry, and surviving supersymmetry is manifest in this superfield formalism. BPS equations for walls also respect this supersymmetry automatically, as we will explain later. On the other hand, the gauge field $W_{\mu}$ vanishes in the BPS background, and is only induced by the order $\lambda$ fluctuations of moduli fields. Since the gauge field appears as the coefficient of $\bar{\theta} \gamma^{\mu} \theta \sim \mathscr{O}\left(\lambda^{-1}\right)$, we find the vector multiplet to be of the order of

$$
\mathbf{V} \sim \mathscr{O}(1), \quad\left(W_{\mu} \sim \mathscr{O}(\lambda)\right)
$$

Neglecting $\mathscr{O}\left(\lambda^{4}\right)$ we obtain

$$
\begin{aligned}
\mathscr{L}= & -\mathscr{E}_{\mathrm{w}}+\int d^{4} \theta \operatorname{Tr}\left[-2 c \mathbf{V}+e^{2 \mathbf{V}} \mathbf{H}^{1} \mathbf{H}^{1 \dagger}+\frac{1}{2 g^{2}}\left(e^{-2 \mathbf{V}} \hat{D}_{y} e^{2 \mathbf{V}}\right)^{2}\right] \\
& +\left(\int d^{2} \theta \operatorname{Tr}\left[\hat{D}_{y} \mathbf{H}^{1} \mathbf{H}^{2 \dagger}-\mathbf{H}^{1} M \mathbf{H}^{2 \dagger}\right]+\text { h.c. }\right) .
\end{aligned}
$$


Up to this order, we can see that $\mathbf{H}^{2}, \mathbf{V}$ serve as Lagrange multiplier fields. Namely the field equations for $\mathbf{H}^{2}$ and $\mathbf{V}$ give constraints

$$
\begin{aligned}
\hat{D}_{y} \mathbf{H}^{1} & =\mathbf{H}^{1} M, \\
g^{2}\left(c-\mathbf{H}^{1} \mathbf{H}^{1 \dagger} e^{2 \mathbf{V}}\right) & =-\hat{D}_{y}\left(e^{-2 \mathbf{V}} \hat{D}_{y} e^{2 \mathbf{V}}\right),
\end{aligned}
$$

respectively. By expanding the superfield constraints in powers of the Grassmann coordinates $\theta, \bar{\theta}$, we find, at the leading order, the BPS equations (2.3), and (2.4) for the hypermultiplet $H^{1}$, and vector multiplet scalar $\Sigma$ with $H^{2}=0$.

We can now choose a convenient gauge of the complexified $U\left(N_{\mathrm{C}}\right)$ local gauge invariance. Let us define an element of the complexified gauge transformation $\mathbf{S}$ to express the chiral scalar superfield $\Phi$ for the adjoint scalar of the vector multiplet as a pure gauge

$$
\Phi=\mathbf{S}^{-1} \partial_{y} \mathbf{S} .
$$

Then the constraint equation (3.13) for the hypermultiplet chiral superfield becomes simpler

$$
\partial_{y}\left(\mathbf{S H}^{1}\right)=\mathbf{S H}^{1} M,
$$

which is easily solved in terms of the moduli matrix chiral superfields $\mathbf{H}_{0}$ as

$$
\mathbf{H}^{1}(x, \boldsymbol{\theta}, \bar{\theta}, y)=\mathbf{S}^{-1}(x, \boldsymbol{\theta}, \bar{\theta}, y) \mathbf{H}_{0}(x, \boldsymbol{\theta}, \bar{\theta}) e^{M y} .
$$

After solving the hypermultiplet constraint equation (3.13), we can now define a vector superfield $\mathbf{\Omega}$ which is invariant under the complexified $U\left(N_{\mathrm{C}}\right)$ gauge transformations

$$
\mathbf{\Omega} \equiv \mathbf{S} e^{-2 \mathbf{V}} \mathbf{S}^{\dagger}
$$

The remaining constraint (3.14) can be rewritten in terms of the gauge invariant superfield $\boldsymbol{\Omega}$ as

$$
\partial_{y}\left(\boldsymbol{\Omega}^{-1} \partial_{y} \boldsymbol{\Omega}\right)=g^{2} c\left(1-\boldsymbol{\Omega}^{-1} \boldsymbol{\Omega}_{0}\right), \quad \mathbf{\Omega}_{0} \equiv c^{-1} \mathbf{H}_{0} e^{2 M y} \mathbf{H}_{0}^{\dagger},
$$

which gives the master equation (2.9) as the lowest component. Therefore this is the superfield extension of the master equation.

By using the solution of the constraint equation (3.13) for the hypermultiplet superfield $\mathbf{H}^{1}$, we can rewrite the fundamental Lagrangian in Eq. 3.12) (up to order $\mathscr{O}\left(\lambda^{2}\right)$ ) in terms of the gauge invariant superfield $\boldsymbol{\Omega}$ as

$$
\mathscr{L}=-\mathscr{E}_{\mathrm{W}}+\int d^{4} \theta\left[c \log \operatorname{det} \boldsymbol{\Omega}+c \operatorname{Tr}\left(\boldsymbol{\Omega}_{0} \boldsymbol{\Omega}^{-1}\right)+\frac{1}{2 g^{2}} \operatorname{Tr}\left(\boldsymbol{\Omega}^{-1} \partial_{y} \boldsymbol{\Omega}\right)^{2}\right]+\mathscr{O}\left(\lambda^{4}\right)
$$

The first, second, and third terms in the $d^{4} \theta$ integrand come from the corresponding terms in the $d^{4} \theta$ integrand of the fundamental Lagrangian (3.12) (up to order $\mathscr{O}\left(\lambda^{2}\right)$ ).

The superspace extension 3.19) of the master equation provides a method to determine all the quantities of interest as a systematic expansion in powers of Grassmann coordinates $\theta, \bar{\theta}$ as follows. Suppose we have an exact solution $\Omega_{\text {sol }}\left(H_{0}, H_{0}^{\dagger}, y\right)$ for the master equation 2.9 ) as a function of moduli matrix $H_{0}, H_{0}^{\dagger}$

$$
\Omega=\left.\boldsymbol{\Omega}\right|_{\theta=0}=\Omega_{\mathrm{sol}}\left(H_{0}(x), H_{0}^{\dagger}(x), y\right)
$$


By promoting the moduli matrix to a superfield $\mathbf{H}_{0}, \mathbf{H}_{0}^{\dagger}$ defined in Eq. 3.17 , we obtain the solution for the vector superfield $\boldsymbol{\Omega}$ of the superfield master equation 3.19 as a composite of the chiral and the anti-chiral superfields,

$$
\Omega_{\mathrm{sol}}\left(\mathbf{H}_{0}(x, \boldsymbol{\theta}), \mathbf{H}_{0}^{\dagger}(x, \overline{\boldsymbol{\theta}}), y\right) \equiv \boldsymbol{\Omega}_{\mathrm{sol}} .
$$

As we noted in Eq.(3.18), the superfield $\boldsymbol{\Omega}=\mathbf{S} e^{-2 \mathbf{V}} \mathbf{S}^{\dagger}$ is $U\left(N_{\mathrm{C}}\right)$ supergauge invariant, but the division between $\mathbf{S},\left(\mathbf{S}^{\dagger}\right)$ and $e^{-2 \mathbf{V}}$ depends on the gauge choice. In obtaining the solution for the fluctuation fields such as $W_{\mu}$, we need to choose the Wess-Zumino gauge for the real general (vector) superfield $\mathbf{V}_{\text {sol }}$. This gauge transformation to the Wess-Zumino gauge is expressed as a multiplication of the chiral $\mathbf{S}_{\mathrm{sol}}$ and anti-chiral $\mathbf{S}_{\mathrm{sol}}^{\dagger}$ superfields from left and right respectively as

$$
\mathbf{S}_{\mathrm{sol}} e^{-2 \mathbf{V}_{\mathrm{sol}}} \mathbf{S}_{\mathrm{sol}}^{\dagger}=\boldsymbol{\Omega}_{\mathrm{sol}} \text {. }
$$

Then expansion of the left-hand side of (3.23) in powers of the Grassmann coordinates $\theta, \bar{\theta}$ gives

$$
\mathbf{S}_{\mathrm{sol}} e^{-2 \mathbf{V}_{\mathrm{sol}}} \mathbf{S}_{\mathrm{sol}}^{\dagger}=S_{\mathrm{sol}} S_{\mathrm{sol}}^{\dagger}+\theta \sigma^{\mu} \overline{\boldsymbol{\theta}}\left(i\left(\partial_{\mu} S_{\mathrm{sol}}\right) S_{\mathrm{sol}}^{\dagger}-i S_{\mathrm{sol}}\left(\partial_{\mu} S_{\mathrm{sol}}^{\dagger}\right)+2 S_{\mathrm{sol}} W_{\mu}^{\mathrm{sol}} S_{\mathrm{sol}}^{\dagger}\right)+\cdots,
$$

where we have not displayed the bilinear terms of fermions, and dots denote other powers of Grassmann coordinates. Expanding the right-hand side of Eq. 3.23) we obtain

$$
\Omega_{\mathrm{sol}}\left(\mathbf{H}_{0}, \mathbf{H}_{0}^{\dagger}, y\right)=\Omega_{\mathrm{sol}}+\theta \sigma^{\mu} \bar{\theta}\left(i\left(\delta_{\mu}-\delta_{\mu}^{\dagger}\right) \Omega_{\mathrm{sol}}\right)+\cdots,
$$

where we have defined the variation $\delta_{\mu}$ and its conjugate $\delta_{\mu}^{\dagger}$ with respect to the scalar fields of chiral superfields and anti-chiral superfields

$$
\delta_{\mu} \equiv \sum_{i} \partial_{\mu} \phi^{i} \frac{\delta}{\delta \phi^{i}}, \quad \delta_{\mu}^{\dagger} \equiv \sum_{i} \partial_{\mu} \phi^{i *} \frac{\delta}{\delta \phi^{i *}},
$$

respectively. If the variation $\delta_{\mu}$ and $\delta_{\mu}^{\dagger}$ act on those functions which depend on the world-volume coordinates $x^{\mu}$ only through moduli fields, they satisfy $\partial_{\mu}=\delta_{\mu}+\delta_{\mu}^{\dagger}$.

Comparing the lowest components of (3.24) and (3.25), we obtain

$$
S_{\mathrm{sol}} S_{\mathrm{sol}}^{\dagger}=\Omega_{\mathrm{sol}} \text {. }
$$

This shows that we cannot avoid $S_{\text {sol }}$ to depend on both $\phi^{i}$ and $\phi^{i *}$, since we cannot factorize these dependences in $\Omega_{\text {sol }}$. One should note that $\mathbf{S}_{\mathrm{sol}}\left(\mathbf{S}_{\mathrm{sol}}^{\dagger}\right)$ is still chiral (anti-chiral) scalar superfield, taking both $\phi^{i}$ and $\phi^{i *}$ as lowest components of chiral scalar superfields. Comparison of the vector components of (3.24) and (3.25), we obtain a solution of the gauge fields as

$$
-i W_{\mu}^{\mathrm{sol}}=S_{\mathrm{sol}}^{-1} \delta_{\mu}^{\dagger} S_{\mathrm{sol}}+S_{\mathrm{sol}}^{\dagger} \delta_{\mu} S_{\mathrm{sol}}^{\dagger-1}+(\text { bi-linear terms of fermions })
$$

It is interesting to observe that this solution of gauge field fluctuation $W_{\mu}^{\text {sol }}$ receives contributions only from the $\phi^{i *}\left(\phi^{i}\right)$ dependence of $S_{\text {sol }}\left(S_{\text {sol }}^{\dagger}\right)$, in spite of the $\mathbf{S}_{\mathrm{sol}}$ being the chiral superfield. Similarly the adjoint scalar $\Sigma$ and the gauge field $W_{y}$ in the extra fifth direction is obtained from the lowest component of Eq. 3.15)

$$
\Phi_{\mathrm{sol}}=\mathbf{S}_{\mathrm{sol}}^{-1} \partial_{y} \mathbf{S}_{\mathrm{sol}} \quad \rightarrow \quad \Sigma^{\mathrm{sol}}+i W_{y}^{\mathrm{sol}}=S_{\mathrm{sol}}^{-1} \partial_{y} S_{\mathrm{sol}}
$$


The other components of the superfields $\boldsymbol{\Omega}, \mathbf{V}$, and $\Phi$ are similarly determined by the superfield equations.

In order to obtain the low-energy effective Lagrangian $\mathscr{L}_{\text {eff }}$, we just need to substitute the solutions $\boldsymbol{\Omega}_{\text {sol }}$ into the fundamental Lagrangian $\mathscr{L}$ and integrate over the extra dimensional coordinate $y$. The resulting four-dimensional effective Lagrangian for $\mathbf{H}_{0}$ is given by

$$
\mathscr{L}_{\text {eff }}=\int d y \mathscr{L}=-T_{\mathrm{w}}+\int d^{4} \theta K\left(\phi, \phi^{*}\right)+\mathscr{O}\left(\lambda^{4}\right),
$$

where the Kähler potential is expressed by an integral form as

$$
K\left(\phi, \phi^{*}\right)=\left.\int d y \mathscr{K}\left(\phi, \phi^{*}, \boldsymbol{\Omega}, y\right)\right|_{\Omega=\Omega_{\mathrm{sol}}},
$$

with a density

$$
\mathscr{K}\left(\phi, \phi^{*}, \boldsymbol{\Omega}, y\right)=c \log \operatorname{det} \boldsymbol{\Omega}+c \operatorname{Tr}\left(\mathbf{\Omega}_{0} \boldsymbol{\Omega}^{-1}\right)+\frac{1}{2 g^{2}} \operatorname{Tr}\left(\mathbf{\Omega}^{-1} \partial_{y} \mathbf{\Omega}\right)^{2} .
$$

Since we are considering the massless fields corresponding to the moduli, we naturally obtain a nonlinear sigma model whose kinetic terms are specified by the Kähler potential $K\left(\phi, \phi^{*}\right)$, without any potential terms. Let us note that our method gives the density of the Kähler potential directly without going through the Kähler metric. This is in contrast to the component approach where one usually obtains the Kähler metric of the nonlinear sigma model with component scalar fields, and then integrate it to obtain the Kähler potential with a lot of labor. It is interesting to note that our effective Lagrangian is not just an effective Lagrangian on a single wall, but an effective Lagrangian on the multiple wall system with various moduli such as relative distance moduli as the effective fields. Therefore we can discuss strings stretched between multiple walls (branes), which was difficult previously as a BIon [14]. ${ }^{3}$

By using the superfield master equation (3.19), we can show that the second term in Eq. 3.32) becomes a total derivative term. Therefore it can be omitted from the effective Lagrangian. The wall tension $T_{\mathrm{w}}$ is given by the topological charge in Eq.(2.5) as an integral over the total derivative term $\mathscr{E}_{\mathrm{w}}$ in Eq. (3.8) by using the boundary condition which requires that vacua are reached at both infinities $y= \pm \infty$.

In the strong coupling limit $g^{2} \rightarrow \infty$, the superfield master equation 3.19 becomes just an algebraic equation $\Omega=\Omega_{0}$, and exact solutions for $\Omega$ can be obtained and the Kähler potential assumes a simple form in this case [8]

$$
K_{0}\left(\phi, \phi^{*}\right)=c \int d y \log \operatorname{det} \Omega_{0}
$$

In [1] we also worked out the integrand of the effective Kähler potential for non-Abelian vortices, whose moduli matrix was established in [15]. It turned out to contain the Wess-ZuminoWitten-like term. On the other hand it has been recently shown that the one for domain wall networks (loops) [16] takes the similar form with [3.32] [17]. Our method should be applicable to monopoles (instantons).

\footnotetext{
${ }^{3}$ We can construct the full solutions of this composite soliton as a 1/4 BPS state 9 .
} 


\section{References}

[1] M. Eto, Y. Isozumi, M. Nitta, K. Ohashi and N. Sakai, Phys. Rev. D 73 (2006) 125008 [arXiv:hep-th/0602289].

[2] P. Horava and E. Witten, Nucl. Phys. B460 (1996) 506 [arXiv:hep-th/9510209]; N. Arkani-Hamed, S. Dimopoulos and G. R. Dvali, Phys. Lett. B429 (1998) 263 [arXiv:hep-ph/9803315]; I. Antoniadis, N. Arkani-Hamed, S. Dimopoulos and G. R. Dvali, Phys. Lett. B436 (1998) 257 [arXiv:hep-ph/9804398]; L. Randall and R. Sundrum, Phys. Rev. Lett. 83 (1999) 3370 [arXiv:hep-ph/9905221]; Phys. Rev. Lett. 83 (1999) 4690 [arXiv:hep-th/9906064].

[3] S. Dimopoulos and H. Georgi, Nucl. Phys. B193 (1981) 150; N. Sakai, Z. f. Phys. C11 (1981) 153; E. Witten, Nucl. Phys. B188 (1981) 513; S. Dimopoulos, S. Raby and F. Wilczek, Phys. Rev. D24 (1981) 1681.

[4] E. Witten and D. Olive, Phys. Lett. 78B (1978) 97.

[5] E. Bogomol'nyi, Sov. J. Nucl. Phys. B24 (1976) 449 [Yad. Fiz. 24 (1976) 861]; M.K. Prasad and C.H. Sommerfield, Phys. Rev. Lett. 35 (1975) 760.

[6] M. Eto, Y. Isozumi, M. Nitta, K. Ohashi and N. Sakai, J. Phys. A 39 (2006) R315 [arXiv:hep-th/0602170].

[7] N. S. Manton, Phys. Lett. B 110 (1982) 54.

[8] Y. Isozumi, M. Nitta, K. Ohashi and N. Sakai, Phys. Rev. Lett. 93 (2004) 161601 [arXiv:hep-th/0404198]; Phys. Rev. D70 (2004) 125014 [arXiv:hep-th/0405194]; M. Eto, Y. Isozumi, M. Nitta, K. Ohashi, K. Ohta and N. Sakai, Phys. Rev. D 71 (2005) 125006 [arXiv:hep-th/0412024]; M. Eto, Y. Isozumi, M. Nitta, K. Ohashi, K. Ohta, N. Sakai and Y. Tachikawa, Phys. Rev. D 71 (2005) 105009 [arXiv:hep-th/0503033].

[9] Y. Isozumi, M. Nitta, K. Ohashi and N. Sakai, Phys. Rev. D 71 (2005) 065018 [arXiv:hep-th/0405129].

[10] J. Wess and J. Bagger, Supersymmetry and Supergravity, Princeton University Press, (1992).

[11] A. Hebecker, Nucl. Phys. B632 (2002) 101 [arXiv:hep-ph/0112230].

[12] K. Kakimoto and N. Sakai, Phys. Rev. D 68 (2003) 065005 [arXiv:hep-th/0306077].

[13] N. Arkani-Hamed, T. Gregoire and J. Wacker, JHEP 0203 (2002) 055 [arXiv: hep-th/0101233];

D. Marti and A. Pomarol, Phys. Rev. D64 (2001) 105025 [arXiv:hep-th/0106256].

[14] C. G. Callan and J. M. Maldacena, Nucl. Phys. B513 (1998) 198 [arXiv:hep-th/9708147]; G. W. Gibbons, Nucl. Phys. B514 (1998) 603 [arXiv:hep-th/9709027].

[15] M. Eto, Y. Isozumi, M. Nitta, K. Ohashi and N. Sakai, Phys. Rev. Lett. 96 (2006) 161601 [arXiv:hep-th/0511088]; M. Eto, T. Fujimori, Y. Isozumi, M. Nitta, K. Ohashi, K. Ohta and N. Sakai, Phys. Rev. D 73 (2006) 085008 [arXiv:hep-th/0601181]; M. Eto, K. Konishi, G. Marmorini, M. Nitta, K. Ohashi, W. Vinci and N. Yokoi, Phys. Rev. D 74 (2006) 065021 [arXiv:hep-th/0607070]; M. Eto, K. Hashimoto, G. Marmorini, M. Nitta, K. Ohashi and W. Vinci, Phys. Rev. Lett. 98 (2007) 091602 [arXiv:hep-th/0609214].

[16] M. Eto, Y. Isozumi, M. Nitta, K. Ohashi and N. Sakai, Phys. Rev. D 72 (2005) 085004 [arXiv:hep-th/0506135]; Phys. Lett. B 632 (2006) 384 [arXiv:hep-th/0508241]; M. Eto, Y. Isozumi, M. Nitta, K. Ohashi, K. Ohta and N. Sakai, AIP Conf. Proc. 805 (2006) 354 [arXiv:hep-th/0509127].

[17] M. Eto, T. Fujimori, T. Nagashima, M. Nitta, K. Ohashi and N. Sakai, Phys. Rev. D 75 (2007) 045010 [arXiv:hep-th/0612003]. 